\title{
Anticonceptivos orales y riesgo de accidente isquémico: meta-análisis
}

Ischemic Stroke Risk With Oral Contraceptives. A Meta-analysis. Gillum LA, Mamidipudi SK, Johnston SC. JAMA $2000 ; 284: 72-78$.

\begin{abstract}
Objetivo
Investigar la bibliografía disponible sobre el incremento del riesgo de accidente isquémico asociado al uso de anticonceptivos orales (ACO).

\section{Diseño}

Meta-análisis.

\section{Fuente y selección de datos}

Realizaron una búsqueda sistemática de toda la literatura publicada desde 1960 hasta noviembre de 1999; usando el Index Medicus (antes de 1966), MEDLINE (después de 1966); BIOIS (después 1985) y Dissertation Abstracts Online (North American Universities). Los términos empleados para la búsqueda fueron: oral contraceptives (side effects, complications), stroke, estrogen, cerebral, ischemic, trombosis, and venous sinus. De 10.409 referencias identificadas, 804 fueron consideradas potencialmente relevantes y de ellas, 73 analizaron el uso de ACO y el riesgo de accidente isquémico (casos controles y estudios de cohortes). De los 73 sólo 16 reunieron los criterios de inclusión. La extracción de los datos fue realizada por dos investigadores independientes, y los desacuerdos lo resolvieron a través de un tercer investigador y mediante discusión.
\end{abstract}

\section{Medición de resultados principales}

Se analizaron 16 estudios. Para el análisis primario utilizaron estimadores de riesgo, controlando por los potenciales confundidores* mediante estratificación, apareamiento o análisis multivariado.

\section{Resultados Principales}

El riesgo estimado global para accidente isquémico, en las usadoras actuales comparada con las no usadoras actuales de ACO, fue de 2.75 (95\% IC, 2.24-3.38, $p<0.001)$. A pequeñas dosis de estrógeno el riesgo fue más bajo $(p=0.01)$, y con dosis altas el riesgo fue significativamente elevado. Los estudios no controlaron por consumo de tabaco $(p=0.01)$, ni por hipertensión $(p=0.04)$ y en aquellos estudios que utilizaron a pacientes hospitalarios el RR fue elevado $(p<0.001)$, El RR de accidentes isquémicos en las usadoras de ACO fue afectado mínimamente por otros factores de riesgo (hipertensión, migraña y edad $\geq 35$ años) y por el diseño del estudio. En los estudios realizados en la población general el RR fue de 1.93 (95\% IC, 1.35-2.74) para preparaciones con bajas dosis de estrógeno, controlados por tabaco e hipertensión. Todo expresado anteriormente, se traduce en un riesgo adicional de 4.1 accidentes isquémico por 100000 mujeres que usan ACO con bajas dosis de estrógeno, no fumadoras y normotensas, 01 accidente isquémico adicional por año cada 24000 mujeres usadoras de ACOs.

\section{Conclusiones}

Los resultados finales indican que el riesgo de accidente isquémico se incrementa en las utilizadoras actuales de ACO, incluso con las nuevas preparaciones bajas en estrógeno. Sin embargo, el riesgo absoluto y la incidencia de accidente isquémico en la población es muy baja.

Fuente de financiamiento: no referida

\section{Comentario}

Los ACO son utilizados por más de 200 millones de mujeres en todo el mundo, y por 1 de cada 4 mujeres menores de 45 años en EE.UU1. Como expresan los autores del estudio los efectos adversos de los ACO se conocen desde su introducción hace 40 años y es desde entonces que la dosis del componente estrogénico fue disminuyendo progresivamente para disminuir tales efectos. Desde la década del 60 hasta la actualidad se realizaron muchos estudios observacionales (cohortes y casoscontroles) para ver la asociación del uso de ACO y el riesgo de accidente isquémico, pero ningún ensayo clínico randomizado. De ahí que todavía exista tanta controversia sobre el tema. El principal motivo es que en los estudios observacionales, la intervención no es seleccionada al azar (expuestos y no expuestos al factor de riesgo, en este caso, los ACO); por lo tanto son susceptibles de muchos sesgos y tienen menor validez a la hora de determinar una asociación.

Muchos médicos y pacientes creen que los ACO producen numerosos efectos adversos, entre ellos riesgo de accidente isquémico. Este estudio muestra que, si bien el uso de ACO aumenta estadísticamente el riesgo de padecer un ACV isquémi- co, el "impacto clínico" y el "impacto poblacional" es muy pequeño (riesgo absoluto), debido a que estamos ante un evento muy infrecuente en mujeres sanas y jóvenes. También se sabe que la tasa de mortalidad en mujeres menores de 40 años que utilizan ACO es inferior a la de las mujeres que no utilizan ningún método anticonceptivo; diferencia atribuida al aumento de la mortalidad asociada al embarazo y sus complicaciones2, 3 . Esta diferencia aún es mayor si consideramos a las mujeres no fumadoras respecto a las fumadores que consumen ACO. Por lo tanto, este pequeño aumento del riesgo (en términos absolutos) de accidente isquémico no cambiará en general la decisión de recomendar $\mathrm{ACO}$, ya que debemos tener en cuenta la mayor morbimortalidad en las mujeres en edad fértil por patologías asociadas al embarazo.

Es importante recordar que cuando las pacientes nos consultan por anticoncepción, debemos conocer cuáles son sus preferencias y preocupaciones, sus expectativas sobre el tema y la presencia de factores de riesgo; además de informarla sobre los beneficios y efectos nocivos de cada uno de los métodos disponibles para que puedan tomar una decisión informada.

\section{Referencias}

1. United Nations. World Contraceptive Use 1998. New York, NY: United Nations; 1999.

2. Henshaw SK. Unintended pregnancy in the United States. Fam Plann Perspect. 1998; 30: 24-29.

3. Miller K, Rosenfield A. Population and women's reproductive health: an international perspective. Annu Rev Public Health. 1996; 17:359-382. 